\title{
Investigations on Growth, Optical and Thermal Properties of Sulphamic Acid Single Crystals
}

\author{
D. Jaishree, ${ }^{1}$ G. Kanchana, ${ }^{2}$ and R. Kesavasamy ${ }^{3}$ \\ ${ }^{1}$ Department of Physics, Sri Ramakrishna Institute of Technology, Coimbatore, Tamil Nadu 641010, India \\ ${ }^{2}$ Department of Physics, Government Arts College, Coimbatore, Tamil Nadu 641018, India \\ ${ }^{3}$ Department of Physics, Sri Ramakrishna Engineering College, Coimbatore, Tamil Nadu 641022, India
}

Correspondence should be addressed to D. Jaishree; jaishree.damu@gmail.com

Received 10 April 2014; Accepted 21 October 2014; Published 25 November 2014

Academic Editor: Ram N. P. Choudhary

Copyright (C) 2014 D. Jaishree et al. This is an open access article distributed under the Creative Commons Attribution License, which permits unrestricted use, distribution, and reproduction in any medium, provided the original work is properly cited.

\begin{abstract}
Single crystals of sulphamic acid of $11 \times 7 \times 3 \mathrm{~mm}^{3}$ dimension were successfully grown by slow evaporation technique. The crystal structure of grown crystals was confirmed by single crystal X-ray diffraction analysis. The presence of functional groups in the crystal lattice has been qualitatively determined by FT-IR and FT-Raman analyses. Theoretical group factor analysis predicts the possible modes of vibrations. The optical transmission spectroscopy (UV-Vis) clearly evidences the suitability of this material for optical application. The thermal behaviour of the crystal was studied by thermogravimetric (TG) and differential scanning calorimetric (DSC) studies. The nonlinear optical (NLO) characteristic of this material was explored by the second harmonic generation (SHG) conversion efficiency.
\end{abstract}

\section{Introduction}

Nonlinear optical (NLO) materials exhibiting second harmonic generation have been in great demand over the last few decades due to technological importance in the fields of optical communication, signal processing, and instrumentation [1-3]. Most of the organic NLO crystals usually have poor mechanical and thermal properties and are susceptible to damage during processing even though they have large NLO efficiency. Also it is difficult to grow larger size optical-quality crystals of these materials for device applications. Most of the recent work has demonstrated that organic crystals can have very large nonlinear susceptibilities compared with inorganic crystals, but their use is impeded by their low optical transparencies, poor mechanical properties, low laser damage thresholds, and inability to produce and process large crystals [4]. The inorganic materials are widely used in these applications because of their high melting point, high mechanical strength, and high degree of chemical inertness [5].

Sulphamic acid $\left(\mathrm{H}_{2} \mathrm{NSO}_{3} \mathrm{H}\right)$ is the monoamide of sulphuric acid and is formed as orthorhombic crystal. It is highly stable and it can be kept for years without any change in properties. It is a strong inorganic acid; while mixing it with water it exhibits Zwitterionic form [1]. Owing to these advantages, JIS (Japanese Industrial Standard) has established this reagent as a standard substance for titrimetric analysis and the British Analytical Methods Committee as well as IUPAC has also recommended the acid [2]. Mainly, solution growth techniques have been applied for growing good quality crystals. The limited thermal stability of many of the compounds examined makes particularly exploratory solution growth as the first technique to be considered [3]. Crystal growth from solution is an important process that is used in many applications from laboratory to industrial scale. The number of organic and inorganic crystals is grown in this fashion [6].

In the present investigation, sulphamic acid (SA) single crystals have been grown successfully by slow evaporation technique. The modes of vibrations were analysed for the first time by the factor group analysis. The grown crystals were characterized by powder X-ray diffraction and single crystal X-ray diffraction analysis, FT-IR, FT RAMAN, and optical transmittance studies. TGA and DSC analysis show 


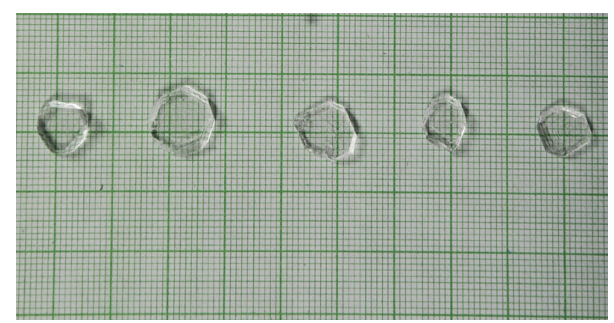

FIGURE 1: Photograph of as-grown SA single crystals.

the thermal behavior of the crystals. The SHG studies for the sulphamic acid single crystal showed it is SHG active as reported in the literature [4].

\section{Growth Procedure}

Saturated solution of SA was prepared using double distilled water at room temperature. The solution is stirred continuously to get homogeneous mixture. It is filtered using Whatman filter paper. The filtered solution was poured in various beakers and was covered by filter paper. Good transparent single crystals were obtained after 12 days. The crystals were purified by the process of repeated recrystallization (see Figure 1).

\section{Characterization Studies}

Single crystal X-ray diffraction was carried out using Enraf Nonius-CAD 4 diffractometer to determine the cell parameters and crystal structure. The FT-IR and FT-Raman spectra were recorded using BRUKER: RFS spectrometer in frequency range $400-4000 \mathrm{~cm}^{-1}$ to analyze the presence of functional groups. The optical transmission spectrum was recorded by double beam UV-Vis spectrophotometer in the range $200-800 \mathrm{~nm}$ covering the entire near ultraviolet, visible, and NIR regions. The NLO property was tested using Kurtz and Perry powder test.

\section{Results and Discussion}

4.1. Single Crystal X-Ray Diffraction Analysis. The titled material has been subjected to single crystal XRD employing Enraf Nonius-CAD 4 diffractometer. The single crystal XRD study reveals that the grown crystal belongs to orthorhombic crystal system and the obtained lattice parameters are $a=$ $8.05 \AA, b=8.17 \AA$, and $c=9.23 \AA$. These values are compared with already reported value of SA [7] crystals and are tabulated in Table 1.

From the XRD analysis, it is confirmed that the grown crystal crystallizes to orthorhombic crystal structure with Pbca space group. The unit cell volume is $596 \AA$. The density is found to be $2.141 \mathrm{mg} / \mathrm{m}^{3}$ and the $Z$ number is 8 .

4.2. FT-IR and FT RAMAN Analysis. Figures 2 and 3 show the recorded spectrum of FT-IR and FT-RAMAN. The spectrum reveals the presence of all functional groups in the

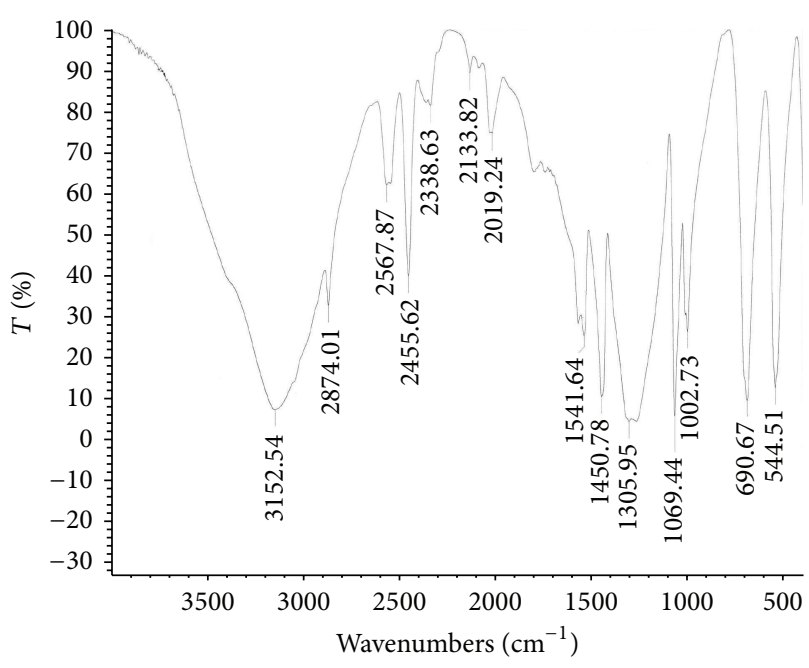

FIGURE 2: FT-IR spectrum.

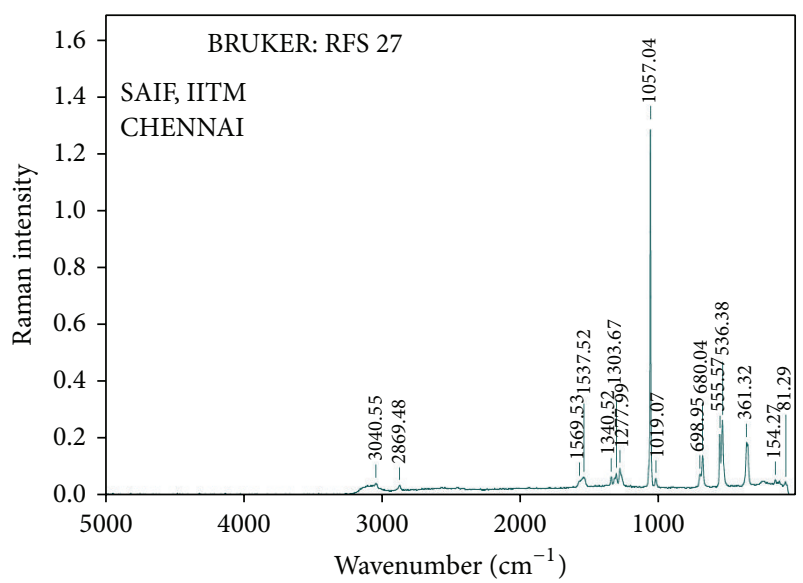

Figure 3: FT-RAMAN spectrum.

crystal. The vibrational assignments for SA single crystal are given in Table 2.

4.3. Factor Group Analysis. A group theoretical analysis predicts the possible modes of vibrations of the materials. The unit cell has 8 atoms which gives rise to $192(3 \times 8$ $\times 8$ ) fundamental modes of vibrations. The factor group analysis of the compound gives rise to 192 normal modes of vibrations distributed as 144 internal and $18\left(\mathrm{~A}_{\mathrm{g}}+\mathrm{B}_{1 \mathrm{~g}}+\right.$ $\left.B_{2 g}+B_{3 g}+A_{u}+B_{1 u}+B_{2 u}+B_{3 u}\right)$ along with 3 acoustical $\left(1 \mathrm{~A}_{\mathrm{g}}+2 \mathrm{~A}_{\mathrm{u}}\right)$ and 45 external modes. The $\mathrm{A}_{\mathrm{g}}$ species are only RAMAN active and $\mathrm{B}_{1 \mathrm{~g}}, \mathrm{~B}_{2 \mathrm{~g}}$, and $\mathrm{B}_{3 \mathrm{~g}}$ species are both IR and RAMAN active. Group theoretical analysis carried out by the procedure outlined by Rosseau et al. [8] shows 3 acoustical, 21 translational, and 24 rotational modes. The summary of factor group analysis is given in Table 3. Each internal mode splits into $\mathrm{B}_{1 \mathrm{~g}}(Z), \mathrm{B}_{2 \mathrm{~g}}(Y), \mathrm{B}_{3 \mathrm{~g}}(X), \mathrm{B}_{1 \mathrm{u}}(Z)$, $\mathrm{B}_{2 \mathrm{u}}(Y)$, and $\mathrm{B}_{3 \mathrm{u}}(X)$ which are IR active and $\mathrm{A}_{\mathrm{g}}(X X, Y Y, Z Z)$, $\mathrm{B}_{1 \mathrm{~g}}(X Y), \mathrm{B}_{2 \mathrm{~g}}(X Z)$, and $\mathrm{B}_{3 \mathrm{~g}}(Y Z)$ are RAMAN active. Table 4 
TABLE 1: Cell parameters of sulphamic acid single crystals.

\begin{tabular}{lcccccccc}
\hline Sample & $a(\AA)$ & $b(\AA)$ & $c(\AA)$ & $\alpha\left(^{\circ}\right)$ & $\beta\left(^{\circ}\right)$ & $\gamma\left(^{\circ}\right)$ & Volume $\left(\mathrm{A}^{3}\right)$ & Crystal system \\
\hline Sulphamic acid & 8.07 & 8.11 & 9.26 & 90 & 90 & 90 & 607.619 & Orthorhombic \\
Sulphamic acid & 8.05 & 8.17 & 9.23 & 90 & 90 & 90 & 596.6 & Orthorhombic \\
\hline
\end{tabular}

TABLE 2: Vibrational assignments.

\begin{tabular}{lcc}
\hline $\begin{array}{l}\text { Wavenumber } \\
\left(\mathrm{cm}^{-1}\right)\end{array}$ & $\begin{array}{c}\text { Wavenumber } \\
\left(\mathrm{cm}^{-1}\right)\end{array}$ & Assignment \\
FTIR & FT RAMAN & \\
\hline 3152 & - & $\mathrm{NH}_{3}^{+}$stretching vibration \\
2874 & 2869 & Symmetric $\mathrm{NH}_{3}{ }^{+}$stretching \\
$2600-2200$ & - & $\mathrm{N}-\mathrm{H}$ stretching \\
1541 & 1537 & Degen. $\mathrm{NH}_{3}{ }^{+}$deformation \\
1450 & - & Symmetric $\mathrm{NH}_{3}{ }^{+}$deformation \\
1305 & 1303 & Asymmetric $\mathrm{SO}_{3}{ }^{-}$stretching \\
1069 & 1057 & Symmetric $\mathrm{SO}_{3}{ }^{-}$deformation \\
1002 & 1019 & Degen. $\mathrm{NH}_{3}{ }^{+}$rocking \\
690 & 680 & $\mathrm{~N}_{-} \mathrm{S} \mathrm{stretching}^{-}$ \\
544 & 555 & Degen. $\mathrm{SO}_{3}{ }^{-}$deformation \\
\hline
\end{tabular}

shows the correlation scheme obtained by the procedure of Fateley et al. [9].

The polarizability tensors are of the forms

$$
\begin{gathered}
\mathrm{A}_{\mathrm{g}}=\left(\begin{array}{ccc}
\alpha_{x x} & 0 & 0 \\
0 & \alpha_{y y} & 0 \\
0 & 0 & \alpha_{z z}
\end{array}\right), \\
\mathrm{B}_{1 \mathrm{~g}}=\left(\begin{array}{ccc}
0 & \alpha_{x y} & 0 \\
0 & 0 & 0 \\
0 & 0 & 0
\end{array}\right), \\
\mathrm{B}_{3 \mathrm{~g}}=\left(\begin{array}{ccc}
0 & 0 & 0 \\
0 & 0 & \alpha_{y z} \\
0 & 0 & 0
\end{array}\right), \\
\mathrm{B}_{2 \mathrm{~g}}=\left(\begin{array}{lll}
0 & 0 & \alpha_{x z} \\
0 & 0 & 0 \\
0 & 0 & 0
\end{array}\right) .
\end{gathered}
$$

4.4. $U V$-VIS-NIR Studies. Molecules containing $\pi$-electrons or nonbonding electrons ( $n$-electrons) can absorb the energy in the form of ultraviolet or visible light to excite these electrons to higher antibonding molecular orbitals. The crystal can be of practical use only when it possesses a good transparency. The optical studies are very important for a crystal since they determine the practical application of the crystal.

The optical transmission spectrum of SA single crystal is shown in Figure 4. The transmittance spectra show that the crystal appears to be transparent in the range $200 \mathrm{~nm}$ to $800 \mathrm{~nm}$.

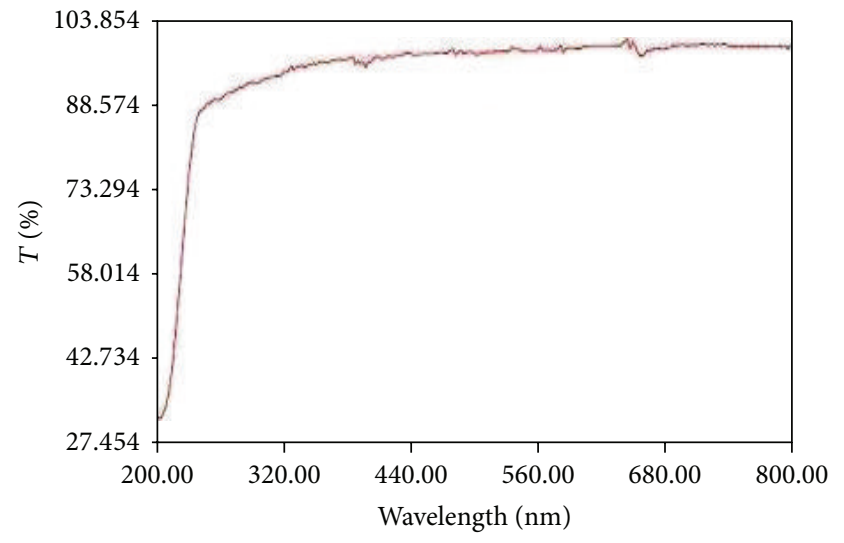

FIGURE 4: Transmittance spectra.

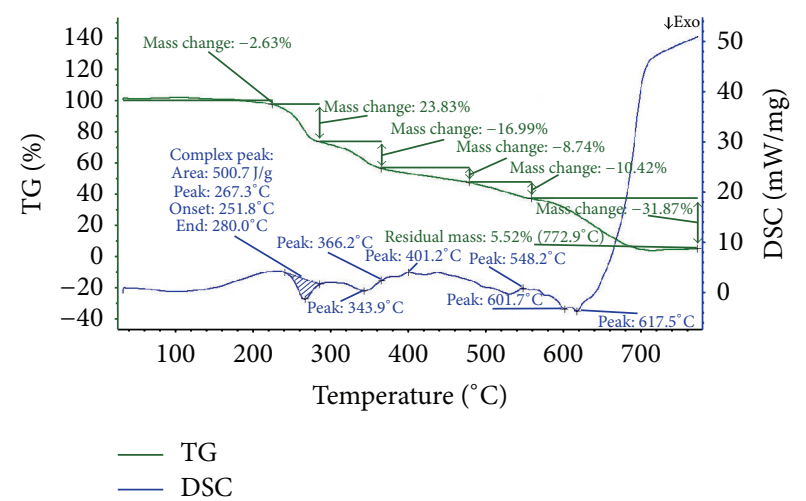

Figure 5: TGA and DSC thermograms.

4.5. Thermogravimetric and Differential Scanning Calorimetric Studies. TGA and DSC thermograms are recorded using NETZSCH STA 409C instrument between room temperature and $700^{\circ} \mathrm{C}$ in nitrogen atmosphere. Simultaneously recorded TGA and DSC thermograms are shown in Figure 5.

The recorded thermogram gives information about the phase transition, water of crystallization, and different stages of decomposition of the crystal. Since the decomposition temperature is beyond $100^{\circ} \mathrm{C}$, there is no evidence for entrapped water in the crystal lattice or any adsorbed water on the crystal surface. The exothermic peak observed at $267^{\circ} \mathrm{C}$ corresponds to the melting point of the crystal. The material is moisture-free and is stable up to $267^{\circ} \mathrm{C}$. This is followed by two exothermic peaks at $366^{\circ} \mathrm{C}$ and $401^{\circ} \mathrm{C}$ which may be due to the decomposition and volatilization of the compound. The total decomposition of the crystal occurs at $617^{\circ} \mathrm{C}$. From the TGA and DSC curves, it is evidenced that the SA crystal is stable up to $267^{\circ} \mathrm{C}$ and can be used for device applications below this temperature. 
TABLE 3: Summary of factor group analysis.

\begin{tabular}{|c|c|c|c|c|c|c|c|c|c|c|}
\hline \multirow{2}{*}{ Factor group symmetry } & \multicolumn{3}{|c|}{ Site symmetry } & \multirow{2}{*}{$\mathrm{S}$} & \multirow{2}{*}{$\mathrm{H}$} & \multirow{2}{*}{$\mathrm{N}$} & \multirow{2}{*}{$\mathrm{O}$} & \multirow{2}{*}{ Optical modes } & \multirow{2}{*}{ Acoustical modes } & \multirow{2}{*}{ Total } \\
\hline & & Ext. & Int. & & & & & & & \\
\hline$\overline{A_{g}}$ & $2 \mathrm{~T}$ & $3 \mathrm{R}$ & 18 & 3 & 9 & 3 & 9 & 24 & 1 & 23 \\
\hline$B_{1 g}$ & $3 \mathrm{~T}$ & $3 \mathrm{R}$ & 18 & 3 & 9 & 3 & 9 & 24 & - & 24 \\
\hline $\mathrm{B}_{2 \mathrm{~g}}$ & $3 \mathrm{~T}$ & $3 R$ & 18 & 3 & 9 & 3 & 9 & 24 & - & 24 \\
\hline $\mathrm{B}_{3 \mathrm{~g}}$ & $3 \mathrm{~T}$ & $3 R$ & 18 & 3 & 9 & 3 & 9 & 24 & - & 24 \\
\hline $\mathrm{A}_{\mathrm{u}}$ & $\mathrm{T}$ & $3 R$ & 18 & 3 & 9 & 3 & 9 & 24 & 2 & 22 \\
\hline $\mathrm{B}_{\text {lu }}$ & $3 \mathrm{~T}$ & $3 R$ & 18 & 3 & 9 & 3 & 9 & 24 & - & 24 \\
\hline $\mathrm{B}_{2 \mathrm{u}}$ & $3 \mathrm{~T}$ & $3 \mathrm{R}$ & 18 & 3 & 9 & 3 & 9 & 24 & - & 24 \\
\hline $\mathrm{B}_{3 \mathrm{u}}$ & $3 \mathrm{~T}$ & $3 \mathrm{R}$ & 18 & 3 & 9 & 3 & 9 & 24 & - & 24 \\
\hline Total & $21 \mathrm{~T}$ & $24 \mathrm{R}$ & 144 & & & & & 192 & 3 & 189 \\
\hline
\end{tabular}

TABLE 4: Correlation scheme.

\begin{tabular}{cccc}
\hline Factor group symmetry & IR active & RAMAN active \\
\hline \multirow{4}{*}{$189 \mathrm{~A}_{\mathrm{g}}$} & - & $\alpha_{x x}, \alpha_{y y}, \alpha_{z z}$ \\
$24 \mathrm{~B}_{\mathrm{gg}}$ & $Z$ & $\alpha_{x y}$ \\
$24 \mathrm{~B}_{2 \mathrm{~g}}$ & $Y$ & $\alpha_{x z}$ \\
$24 \mathrm{~B}_{3 \mathrm{~g}}$ & $X$ & $\alpha_{y z}$ \\
$22 \mathrm{~A}_{\mathrm{u}}$ & - & - \\
$24 \mathrm{~B}_{1 \mathrm{u}}$ & $Z$ & - \\
$24 \mathrm{~B}_{2 \mathrm{u}}$ & $Y$ & - \\
$24 \mathrm{~B}_{3 \mathrm{u}}$ & $X$ & - \\
\hline
\end{tabular}

4.6. Nonlinear Optical Studies. The second harmonic generation efficiency of sulphamic single crystals is measured by using Kurtz-Perry powder SHG technique with potassium dihydrogen phosphate (KDP) crystal as reference material. The crystal was powdered of size ranging from 200 to $250 \mu \mathrm{m}$ and is sandwiched between the glass slides of dimension $7.5 \mathrm{~cm} \times 2.5 \mathrm{~cm} \times 1.3 \mathrm{~mm}$. The fundamental laser beam of $1064 \mathrm{~nm}$ wavelength and input power $0.68 \mathrm{~J}$ was made to fall normally on the sample cell. A photomultiplier tube was used as detector. The output from the sample was monochromated to collect the intensity of $532 \mathrm{~nm}$ component and to eliminate the fundamental radiation. The wavelength of the input radiation has been reduced to half of its initial value. This confirms the frequency doubling which strongly evidences the second harmonic generation of the crystal. The noncentrosymmetric nature of the crystal was also confirmed by its space group in XRD studies. The melting point of the crystal was determined using melting point apparatus and it was found to be $160^{\circ} \mathrm{C}$. Hence there is no possibility of melting of the organic part of the compound when it is exposed to NdYAG laser source. The SHG efficiency of the crystal is found to be 0.89 times that of the standard KDP crystal.

\section{Conclusion}

Single crystals of sulphamic acid have been grown successfully by slow evaporation technique. Single crystal XRD study reveals the structural details of the crystal. The FT-IR and FTRaman spectra show the presence of amine and sulphonic groups. The theoretical factor group analysis predicts 192 vibrational optical modes, which decompose into $\Gamma_{\text {total }}=18$ $\left(A_{g}+B_{1 g}+B_{2 g}+B_{3 g}+A_{u}+B_{1 u}+B_{2 u}+B_{3 u}\right)$ modes with 45 external modes and three acoustical modes $\Gamma_{\text {acou }}=1 A_{g}+2 A_{u}$. The UV-Vis studies showed that the transparent window is good enough in the visible region which is desirous property for this material for NLO applications. The melting point of the crystal was found to be $267^{\circ} \mathrm{C}$. The SHG nature of the crystal was tested by Kurtz and Perry powder testing which is attributed to the noncentrosymmetric nature of the crystals. Its efficiency is found to be 0.89 times that of the standard KDP crystal.

\section{Conflict of Interests}

The authors declare that there is no conflict of interests regarding the publication of this paper.

\section{Acknowledgments}

The authors thank SAIF-IITM for rendering the characterisation facilities. They also extend their thanks to Dr. R. Anandhi for her help in factor group analysis. The authors are dutybound to thank Dr. R. Basheer Ahamed for his help in NLO measurements.

\section{References}

[1] F. A. Kanda and A. J. King, "The crystal structure of sulfamic acid," Journal of the American Chemical Society, vol. 73, no. 5, pp. 2315-2319, 1951.

[2] T. Yoshimori and T. Tanaka, "Preparation of sulphamic acid single crystals and their assay by precise coulometric titration," Analytica Chimica Acta, vol. 66, no. 1, pp. 85-91, 1973.

[3] P. Kerkoc and J. Hulliger, "Growth and characterization of 4(n,n-dimethylamino)-3-acetamidonitrobenzene bulk crystals and single crystal cored fibers," Journal of Crystal Growth, vol. 99, no. 1, pp. 1023-1027, 1990.

[4] M. Lenin, N. Balamurugan, and P. Ramasamy, "Growth and characterization of sulphamic acid single crystals grown by Sanakaranarayanan-Ramasamy (SR) method," Crystal Research and Technology, vol. 42, no. 1, pp. 39-43, 2007. 
[5] F. Zernike and J. E. Midwinter, Applied Nonlinear Optics, John Wiley \& Sons, New York, NY, USA, 1973.

[6] S. A. De Vries, P. Goedtkindt, W. J. Huisman et al., "X-ray diffraction studies of potassium dihydrogen phosphate (KDP) crystal surfaces," Journal of Crystal Growth, vol. 205, no. 1, pp. 202-214, 1999.

[7] H. Pan, X. Gao, Y. Zhang, P. N. Prasad, B. Reinhardt, and R. Kannan, "A new class of heterocyclic compounds for nonlinear optics," Chemistry of Materials, vol. 7, no. 5, pp. 816-821, 1995.

[8] D. L. Rosseau, R. P. Bauman, and S. P. S. Porto, "Normal mode determination in crystals," Journal of Raman Spectroscopy, vol. 10, no. 1, pp. 253-290, 1981.

[9] W. G. Fateley, F. R. Dollish, N. T. MeDevitt, and F. F. Bentley, Infrared and Raman Selection Rules for Molecular and Lattice Vibrations: The Correlation Method, John Wiley \& Sons, New York, NY, USA, 2001. 

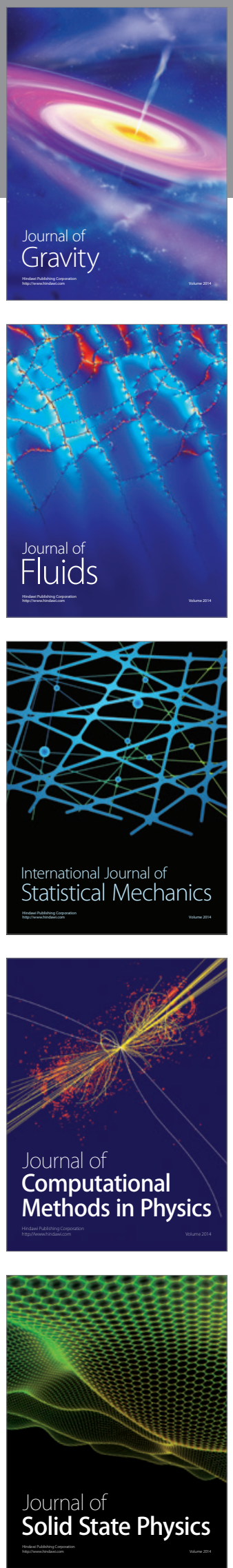

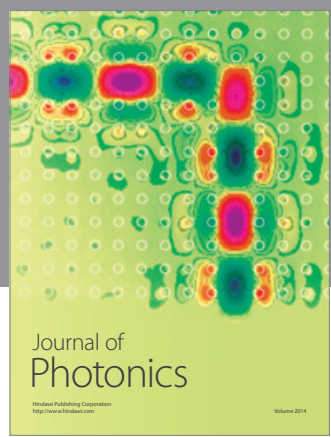

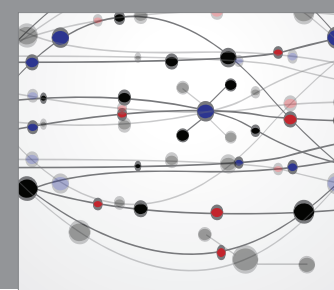

The Scientific World Journal

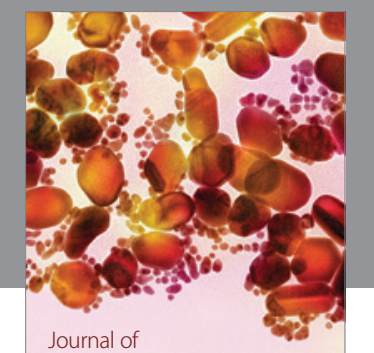

Soft Matter
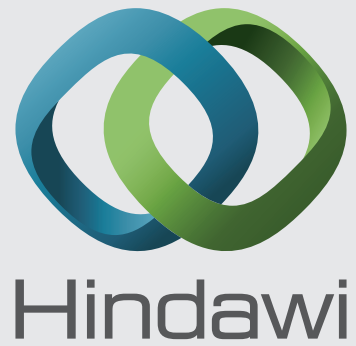

Submit your manuscripts at

http://www.hindawi.com
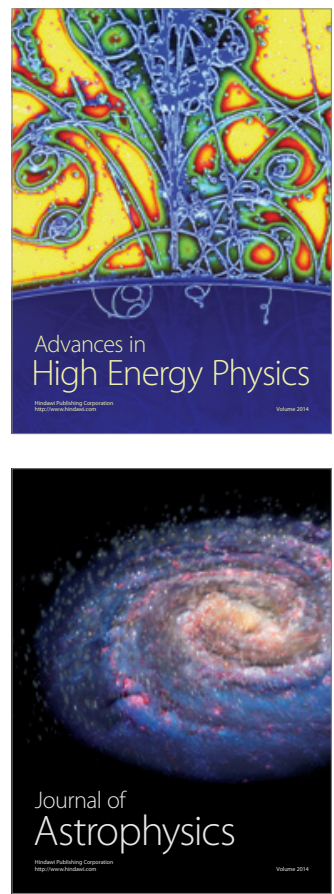
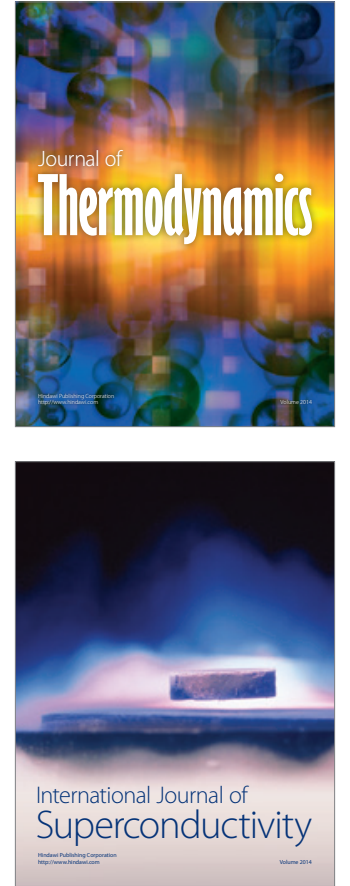
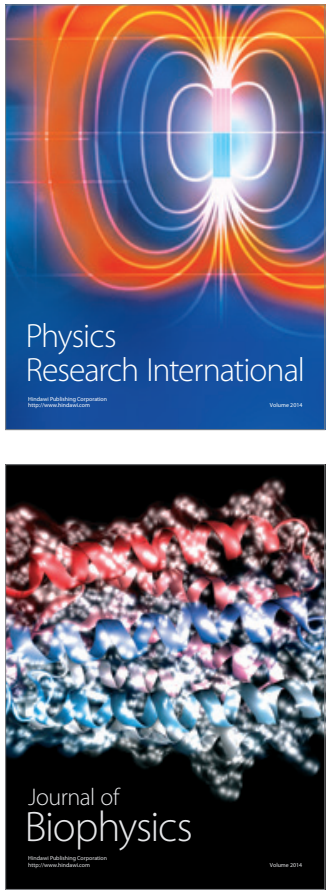
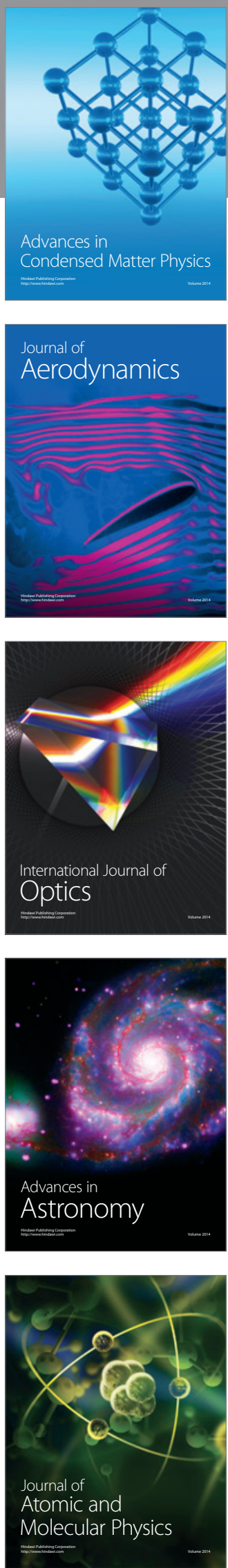